\title{
High-flow nasal cannula oxygen therapy in infants with acute lower respiratory tract infection. An experience in hospitals of the City of Buenos Aires
}

\author{
Ezequiel Monteverde, M.D. ${ }^{a}$, Analía Fernández, M.D. ${ }^{b}$, Fernando Ferrero, M.D. ${ }^{c}$, \\ Cristian Barbaro, M.D. ${ }^{a}$, Leonardo De Lillo, M.D. ${ }^{c}$, Mariana Lavitola, M.D. ${ }^{b}$ and \\ Ariel Golubicki, M.D. ${ }^{d}$ \\ Collaborators: Héctor Cairoli, Edgardo Checacchi, Gustavo Debaisi, Hernán Gaviño, \\ Emiliano Gigliotti, Patricia Landa, Claudia Meregalli, Pablo Neira, Miriam Peralta, \\ Javier Potasznik, Ana Belén Reiner, Alejandra Retta, Julián Rodríguez Kibrik, \\ Guillermo Schwartz, and Claudio Zeltman.
}

\begin{abstract}
Introduction. During the winter, infants with acute lower respiratory tract infection (ALRTI) overburden health resources. In the Autonomous City of Buenos Aires, 35000 children are seen at the hospitals every year; $8-10 \%$ of them are admitted to the general hospitalization ward and $5-12 \%$ of these, to the intensive care unit (ICU). In 2017, the Department of Maternal and Child Health of the Autonomous City of Buenos Aires included high flow nasal cannula (HNFC) oxygen therapy in the ALRTI protocol in the general ward of 3 hospitals. The objective of this study was to describe its outcomes and explore the potential factors related to therapeutic failure.

Methods. Prospective, descriptive study with infants $<18$ months old hospitalized due to ALRTI in 3 hospitals (Durand, Elizalde, Gutiérrez) between June and September 2017. All children unable to comply with low-flow therapeutic targets received HNFC oxygen therapy; admission to the ICU was considered a failure.
\end{abstract}

a. Hospital de Niños Ricardo Gutiérrez.

b. Hospital General de Agudos Carlos Durand.

c. Hospital de Niños Pedro de Elizalde.

d. Department of Maternal and Child Health, Ministry of Health.

City of Buenos Aires.

E-mail address:

Ezequiel

Monteverde, M.D.:

ezequielmonteverde@

gmail.com

Funding:

None

Conflict of interest:

None.

Received: 10-9-2018

Accepted: 4-17-2019 to ALRTI, $39.7 \%$ required HNFC oxygen therapy. No significant baseline differences were observed between patients receiving HNFC and conventional oxygen therapy.

Failure was observed in only $8.7 \%$ of patients with HNFC oxygen therapy. The decrease in respiratory rate was significantly greater and longer in patients with support success versus those with failure $(p<0.01)$. No complications were recorded.

Conclusion. The implementation of HNFC oxygen therapy under a protocol in the general wards was a safe measure. Patients with therapeutic failure showed a smaller reduction in respiratory rate during treatment.

Keywords: bronchiolitis, respiratory tract infections, oxygen therapy, respiratory insufficiency.

dx.doi.org/10.5546/aap.2019.eng.286

To cite: Monteverde E, Fernández A, Ferrero F, Barbaro C, et al. High-flow nasal cannula oxygen therapy in infants with acute lower respiratory tract infection. An experience in hospitals of the City of Buenos Aires. Arch Argent Pediatr 2019;117(5):286-293.

\section{INTRODUCTION}

Pediatric patients with acute lower respiratory tract infection (ALRTI) strongly increase the demand for health care during the winter season, with bronchiolitis being the main clinical entity. ${ }^{1}$ In the Autonomous City of Buenos Aires (CABA), approximately 35000 patients with ALRTI are seen each year and included in the Brief Hospitalization Program, which depends on the public health system. ${ }^{2}$ Between $8 \%$ and $10 \%$ of these patients are admitted to the pediatric general hospitalization ward (PGHW); of these, 5\%-12\% require mechanical ventilation (MV), which accounts for a $40 \%$ bed occupancy in pediatric intensive care units (PICUs) between May and September. ${ }^{3}$

One of the pillars of treatment in these patients is oxygen therapy, both with low-flow (LF) and highflow (HF) systems. HF is defined as the administration of more than $2 \mathrm{~L} /$ min (newborns), $4 \mathrm{~L} / \mathrm{min}$ (infants and children), and $6 \mathrm{~L} / \mathrm{min}$ (adults). A high-flow nasal cannula (HFNC) is an open system that delivers HF oxygen with optimal temperature and relative humidity. Its main action mechanisms include flushing the nasopharyngeal dead space, delivering flow according to the increased inspiratory demand, delivering a measurable fraction of inspired oxygen $\left(\mathrm{FiO}_{2}\right)$, and generating continuous positive airway pressure. ${ }^{4}$

HFNC use has been described in several settings in both newborn infants and children. Observational 
and experimental studies have demonstrated a reduction in the admission rate to the $\mathrm{PICU}^{5-9}$ and $\mathrm{MV}$ requirement $\mathrm{t}^{10-12}$ associated with HFNC use in ALRTI patients. Therefore, in addition to a better tolerance and a lower cost compared to non-invasive mechanical ventilation (NIMV), support with HFNC appears increasingly more interesting. However, few PGHWs use it.

In 2017, the Department of Maternal and Child Health of the Ministry of Health of the Autonomous City of Buenos Aires included support with HFNC in the management of patients with ALRTI in the PGHWs of 3 hospitals. The decision was based on the fact that this strategy may improve the course of these patients, which would reduce the proportion of patients requiring admission to the PICU. For its implementation, a specific protocol was designed (see additional material [AM] in the Annex]. The objective of this report was to describe the outcomes of such implementation and explore the potential factors related to therapeutic failure.

\section{MATERIALS AND METHODS}

Design. Multicenter, prospective, and descriptive study.

Settings. Hospital General de Agudos Carlos Durand (HD) (PGHW and PICU), Hospital General de Niños Pedro de Elizalde (HE) (PGHW 4 and 5, and PICU), and Hospital General de Niños Ricardo Gutiérrez (HG) (PGHW 7 and 8, and PICU).

Duration. Between June $3^{\text {rd }}, 2017$ and September $9^{\text {th }}, 2017$ (epidemiological weeks 22-39).

Population. Infants aged 30 days to 18 months, diagnosed with ALRTI as per the diagnosis and management guidelines established by the Ministry of Health of the $\mathrm{CABA}^{2}$ and hospitalized in the selected PGHWs. The following patients were excluded from use: 1) patients who, upon admission, had uncontrolled or severe disease (hemodynamic instability, inability to protect the airways) and 2) patients with chronic oxygen dependence or cyanotic congenital heart disease.

Work plan. Before the protocol implementation, the health team members of selected PGHWs received theoretical-practical training from experienced health care providers, who helped to design the HFNC use guidelines, included in the protocol by the Ministry of Health mentioned above. The patient selection algorithm was included in these guidelines and a printout was hanged in a visible place inside the participating PGHWs and PICUs.
In accordance with these guidelines, HFNC use was indicated in the case of failure of LF oxygen therapy (up to $3 \mathrm{~L} / \mathrm{min}$ ), established as follows:

1. Inability to achieve oxygen saturation $\left(\mathrm{SaO}_{2}\right)$ by pulse oximetry $\geq 94 \%$ (target $\mathrm{SaO}_{2}$ ).

2. Heart rate (HR) and respiratory rate (RR) above cut-off values after starting LF oxygen therapy and adequate medical treatment or at some point during the course of therapy. Cutoff values were established by age range. ${ }^{13}$ Patients $\leq 6$ months old: $\mathrm{HR} \geq 140 / \mathrm{min}$ and $\mathrm{RR} \geq 55 / \mathrm{min}$; patients $>6$ months old: $H R \geq 140 / \mathrm{min}$ and $R R \geq 45 / \mathrm{min}$.

3. Increased breathing work during the course of therapy as per the treating team's criteria. HF oxygen therapy was administered using the Airvo $2^{\circledR}$ humidifier by Fisher \& Paykel Healthcare ${ }^{\circledR}$ with an Optiflow ${ }^{\circledR}$ infant nasal cannula (up to $20 \mathrm{~L} / \mathrm{min}$ ). The initial configuration was a flow of $2 \mathrm{~L} / \mathrm{kg} / \mathrm{min}$ and a $\mathrm{FiO}_{2}$ of 0.9 , then reduced to less than 0.6 in the first hour and after this, by 0.05 intervals based on the target $\mathrm{SaO}_{2}$, with a constant flow maintained at $2 \mathrm{~L} / \mathrm{kg} / \mathrm{min}$. If the target $\mathrm{SaO}_{2}$ was not achieved, the $\mathrm{FiO}_{2}$ was increased to the previous level. HFNC oxygen therapy was discontinued after maintaining the target $\mathrm{SaO}_{2}$ with a $0.21 \mathrm{FiO}_{2}$ for at least 4 hours.

It was suggested to perform medical treatment in accordance with the referenced guidelines, leaving out the recommendations on feeding for patients on HFNC, which depended on the treating team's judgment.

The following data about all patients with ALRTI were recorded online: date of admission and of hospital discharge, age, weight, sex, reason for admission, presence of complex chronic conditions (CCCs), ${ }^{14}$ identified microorganism, characteristics of chest X-ray upon admission, as per the treating team, classified into 4 patterns. The following data were recorded about the patients who used HFNC: criteria for initiation, date and time of initiation of HFNC, HR and $\mathrm{RR}$ upon admission and at standard hours, and complications. If required, date and time of admission to the PICU and type of additional ventilatory support were also recorded. Two individuals were designated as responsible coordinators at each site and at least 1 person was asked to record data at each hospitalization unit.

Support success (SS) was defined if the patient required only HFNC oxygen therapy at the PGHW, whereas support failure (SF) was 
considered in the following cases: 1) transfer to the PICU or intermediate care unit, 2) NIMV or invasive mechanical ventilation (IMV) requirement.

Ethical considerations: The study was assessed by a research ethics committee and registered before the Central Research Ethics Committee of the Autonomous City of Buenos Aires (MSGCBA) under number $281 / 17$. No informed consent was obtained because this treatment modality was considered the standard of care in the participating units. ${ }^{2}$

Data analysis. Numerical outcome measures were described as mean or median and standard deviation or interquartile range (IQR), based on the adjustment to normality (verified using the Kolmogorov-Smirnov test). Categorical outcome measures were expressed as count and proportion $(\%)$. Proportions were compared using the $\chi^{2}$ test with Yates' correction or Fisher's test for categorical outcome measures, as applicable, and differences between means or medians were verified using Student's t test or the Wilcoxon rank test (WRT). The comparison among more than 2 groups for categorical outcome measures was done using the Kruskal-Wallis test. Based on certain recent controversies, ${ }^{15}$ and for cases that the treating team considered controversial, Bayesian methods were used, ${ }^{16,17}$ as well as those proposed for contingency tables by Jamil et al., ${ }^{18}$ assuming a Poisson distribution. Data were analyzed using the $\mathrm{R}$ free software for Macintosh, version 3.3.3 (2017-3-6, "Another canoe").

\section{RESULTS General}

During the recording period, 522 patients diagnosed with ALRTI were admitted; 207 $(39.7 \%)$ of them required HFNC, and $19(3.6 \%)$ resulted in SF. The patients hospitalized in these PGHWs accounted for $100 \%, 80 \%$, and $57.3 \%$ of admissions due to this cause at $\mathrm{HD}, \mathrm{HG}$, and $\mathrm{HE}$, respectively. The proportion of subjects who were treated with HFNC oxygen therapy varied greatly among the participating PGHWs: $25 \%$ (HD), $40 \%$ (HE-U4), $41 \%$ (HG-U7), $42 \%$ (HE-U5), and $45 \%$ (HG-U8). No significant differences were observed between the patients who had SS with LF oxygen therapy and those who required HFNC in terms of age, weight, sex, reason for admission or presence of CCCs. Although the proportion of patients with congenital heart disease who required HFNC doubled the one that received only LF oxygen therapy (6\% versus $3 \%$ ), such difference was not statistically significant. More microorganisms were identified in the patients who required HFNC (57 \% versus $40 \%$ ); respiratory syncytial virus (RSV) was the most common one. A lower percentage of patients who had a chest X-ray upon admission required HFNC (3\% versus $11 \%$, $p<0.01)$ (Table 1). Some baseline characteristics varied among the 3 hospitals (Table 2).

\section{Characteristics of the population who used high-flow nasal cannula}

All patients who were indicated to use HFNC met at least 1 HFNC initiation criterion, as established by the guidelines (Table 3 ), and more than $90 \%$ met at least 1 of the first 2 .

No statistically significant differences were observed in the time elapsed between admission and HFNC initiation between the SS and groups: 2 hours (0.5-14.1) versus 0.9 hours (0.5-4.3), $p=0.175$ (WRT). The time elapsed between admission and SF was 20.6 hours (13-84). Support with HFNC lasted 98.3 hours (66-147) in the SS group.

The overall length of stay was 4.8 days (3.36.9). The length of stay of patients who required HFNC was 5.9 days (3.9-8.1): 5.9 days (4.9-8.5) in the SS group and 10.4 days (7.6-26.4) in the SF group.

A decrease in the RR was observed in all patients since the initiation of the support with HFNC; however, such reduction was maintained over time in the SS group, whereas it was only seen at the time of initiation in the SF group (Figure 1). The intra-group delta RR was statistically significant ( $t$ test for repeated measures) in the first 4 assessments for the SS group, whereas only in the first comparison in the SF group (Table 4). RR comparisons between both groups (SS versus SF) also showed statistically significant and increasing differences in each control since the first hour (Table 4).

\section{Treatment escalation}

Only 19 of 207 patients who required HFNC ( $8.7 \%$ ) had SF (admission to the PICU): 2 continued with HFNC and 8 received NIMV (successful in 7 cases). NIMV duration (median, IQR) was 43 hours (33-72) (Figure 2). Ten patients required IMV, which accounted for $52.6 \%$ of SF, $4.8 \%$ of HFNC, and $1.9 \%$ of all admitted patients.

The duration of IMV was 10 days (5-13). The length of stay in the PICU depended on the implemented strategies: the patients with IMV 
as the only support $(\mathrm{n}=3)$ were hospitalized for 10.5 days (5-16); those who continued with HFNC and required IMV $(\mathrm{n}=6)$, for 13 days $(10-16)$; and the only patient who required both NIMV and IMV, for 15.5 days. No deaths were recorded across the population sample.

\section{Safety}

No complications associated with HFNC use were observed in this study. Patients were fed orally or through a nasogastric tube and no associated adverse events were reported.

Table 1. Baseline characteristics of patients with and without high-flow nasal cannula

\begin{tabular}{|c|c|c|c|}
\hline With & out HFNC $(n=315)$ & With HFNC $(n=207)$ & $p$ \\
\hline Age (months), med. (IQR) & $6.0(3.0-11.0)$ & $6.0(3.0-10.0)$ & NS \\
\hline Male sex, $\mathrm{n}(\%)$ & $194(62)$ & $123(59)$ & NS \\
\hline \multicolumn{4}{|l|}{ Weight (kg), med. (IQR) } \\
\hline Z-score for weight ${ }^{*}$ (points), & $7.0(5.9-9.0)$ & $7.0(6.0-8.8)$ & NS \\
\hline mean $+/-\mathrm{SD}$ & $-0.96 \pm 1.76$ & $-1.06 \pm 1.60$ & NS \\
\hline \multicolumn{4}{|l|}{ Reason for admission } \\
\hline Bronchiolitis & $202(64)$ & $131(63)$ & NS \\
\hline Bronchospasm & $56(18)$ & $39(19)$ & NS \\
\hline Pneumonia & $51(16)$ & $34(16)$ & NS \\
\hline Pertussis-like syndrome & $6(2)$ & $3(1)$ & NS \\
\hline \multicolumn{4}{|l|}{ Complex chronic conditions } \\
\hline At least one, $\mathrm{n}(\%)$ & $76(24)$ & $68(33)$ & NS \\
\hline Number, med. (IQR) & $0(0-0)$ & $0(0-1)$ & NS \\
\hline Respiratory, n (\%) & $53(17)$ & $43(21)$ & NS \\
\hline Cardiovascular, n (\%) & $11(3)$ & $13(6)$ & NS \\
\hline Neuromuscular, n (\%) & $3(1)$ & $2(1)$ & NS \\
\hline Immune, $\mathrm{n}(\%)$ & $7(2)$ & $2(1)$ & NS \\
\hline Neurological, n (\%) & $11(3)$ & $7(3)$ & NS \\
\hline Genetic, n $(\%)$ & $5(2)$ & $12(6)$ & NS \\
\hline Metabolic, n (\%) & $0(0)$ & $1(0)$ & NS \\
\hline Prematurity, n (\%) & $10(3)$ & $9(4)$ & NS \\
\hline Gastrointestinal, n (\%) & $2(1)$ & $0(0)$ & NS \\
\hline Other, $\mathrm{n}(\%)$ & $1(0)$ & $3(1)$ & NS \\
\hline \multicolumn{4}{|l|}{ Microorganism rescue } \\
\hline Identified microorganism, n (\%) & $127(40)$ & $119(57)$ & $<0.001$ \\
\hline Number (n), med. (IQR) & $0(0-1)$ & $1(0-1)$ & 0.011 \\
\hline Multiple rescue, n (\%) & $1(0)$ & $3(1)$ & NS \\
\hline $\mathrm{RSV}, \mathrm{n}(\%)$ & $94(30)$ & $94(45)$ & $<0.001$ \\
\hline Influenza, $\mathrm{n}(\%)$ & $9(3)$ & $3(1)$ & NS \\
\hline H. influenzae type B, n (\%) & $0(0)$ & $1(0)$ & NS \\
\hline S. pneumoniae, $\mathrm{n}(\%)$ & $0(0)$ & $1(0)$ & NS \\
\hline Adenovirus, $\mathrm{n}(\%)$ & $3(1)$ & $4(2)$ & NS \\
\hline Parainfluenza, n (\%) & $14(4)$ & $16(8)$ & NS \\
\hline Metapneumovirus, n (\%) & $8(3)$ & $2(1)$ & NS \\
\hline B. pertussis, $\mathrm{n}(\%)$ & $1(0)$ & $0(0)$ & NS \\
\hline M. pneumoniae, n (\%) & $0(0)$ & $1(0)$ & NS \\
\hline \multicolumn{4}{|l|}{ Chest X-ray } \\
\hline Pathological, n (\%) & $281(89)$ & $200(97)$ & 0.003 \\
\hline Normal, n $(\%)$ & $34(11)$ & $7(3)$ & 0.004 \\
\hline Hyperinflation of the lungs, $\mathrm{n}(\%)$ & $138(44)$ & $111(54)$ & 0.035 \\
\hline Interstitial infiltrate, $\mathrm{n}(\%)$ & $89(28)$ & $50(24)$ & NS \\
\hline Single infiltrate/ area of condensation, $\mathrm{n}(\%)$ & $44(14)$ & $21(10)$ & NS \\
\hline Multiple infiltrates / areas of condensation, $\mathrm{n}(\%)$ & $6(2)$ & $11(5)$ & NS \\
\hline Atelectasis, $\mathrm{n}(\%)$ & $4(1)$ & $7(3)$ & NS \\
\hline
\end{tabular}

* Calculated based on the Sociedad Argentina de Pediatría. National Committee for Growth and Development. Guideline for the Assessment of Physical Growth. SAP: 2013.

HFNC: high-flow nasal cannula; med.: median; IQR: interquartile range; kg: kilograms; SD: standard deviation; RSV: respiratory syncytial virus; NS: not significant. 


\section{DISCUSSION}

In this sample of patients with ALRTI hospitalized in the pediatric general wards where the HFNC protocol was implemented, such measure was observed to be safe. The sustained improvement of RR after the first hour of support with HFNC is a clear prognostic factor for the success of this method.
The rate of admission to the PICU was $3.6 \%$, lower than that reported for the same year in all children's hospitals as per the Observatory of Acute Lower Respiratory Tract Diseases, dependent on the Ministry of Health of the CABA $(5.1 \%)$. In turn, such rate was also lower than that reported by the same institution for the 20142016 three year period $(8.5 \%)$; however, there are

TABLE 2. Baseline characteristics that differed among the three hospitals

\begin{tabular}{|c|c|c|c|c|}
\hline & $\begin{array}{c}\text { H. Durand } \\
(\mathrm{n}=83,15.9 \%)\end{array}$ & $\begin{array}{c}\text { H. Elizalde } \\
(\mathrm{n}=214,40.9 \%)\end{array}$ & $\begin{array}{c}\text { H. Gutiérrez } \\
(\mathrm{n}=225,43.1 \%)\end{array}$ & $p$ \\
\hline RA: bronchiolitis & $52(63)$ & $166(78)$ & $115(51)$ & $<0.001$ \\
\hline RA: bronchospasm & $16(19)$ & $8(4)$ & $71(32)$ & $<0.001$ \\
\hline $\mathrm{CCC}$ (at least 1$), \mathrm{n}(\%)$ & $19(23)$ & $35(16)$ & $90(40)$ & $<0.001$ \\
\hline Respiratory CCC, n (\%) & $11(13)$ & $8(4)$ & $77(34)$ & $<0.001$ \\
\hline Genetic CCC, $\mathrm{n}(\%)$ & $1(1)$ & $5(2)$ & $11(5)$ & 0.059 \\
\hline Pathological X-ray, n (\%) & $51(61)$ & $209(98)$ & $221(98)$ & $<0.001$ \\
\hline X-ray: hyperinflation of the lungs, $\mathrm{n}(\%)$ & $25(30)$ & $100(47)$ & $124(55)$ & $<0.001$ \\
\hline
\end{tabular}

*RA: reason for admission; CCC: complex chronic condition; X-ray: chest X-ray.

TABLE 3. Physiological outcome measures in patients receiving high-flow nasal cannula oxygen therapy by age range

\begin{tabular}{lcc} 
Outcome measure & $<=\mathbf{6}$ months old $(\mathbf{n}=\mathbf{1 0 2})$ & $>\mathbf{6}$ months old $(\mathbf{n}=\mathbf{9 1})$ \\
\hline $\mathrm{RR}$, cycles $/ \min ($ mean, $95 \% \mathrm{CI})$ & $63(61-65)$ & $63(60-65)$ \\
$\mathrm{HR}$, cycles/min $($ mean, $95 \% \mathrm{CI})$ & $158(155-162)$ & $154(150-159)$ \\
$\mathrm{SaO}_{2}, \%$ (median, IQR) & $96(90-98)$ & $96(92-98)$ \\
\hline
\end{tabular}

RR: respiratory rate; HR: heart rate; $\mathrm{CI}$ : confidence interval; $\mathrm{SaO}_{2}$ : arterial oxygen saturation; IQR: interquartile range.

FIGURE 1. Progression of physiological parameters in the patients with support success and failure

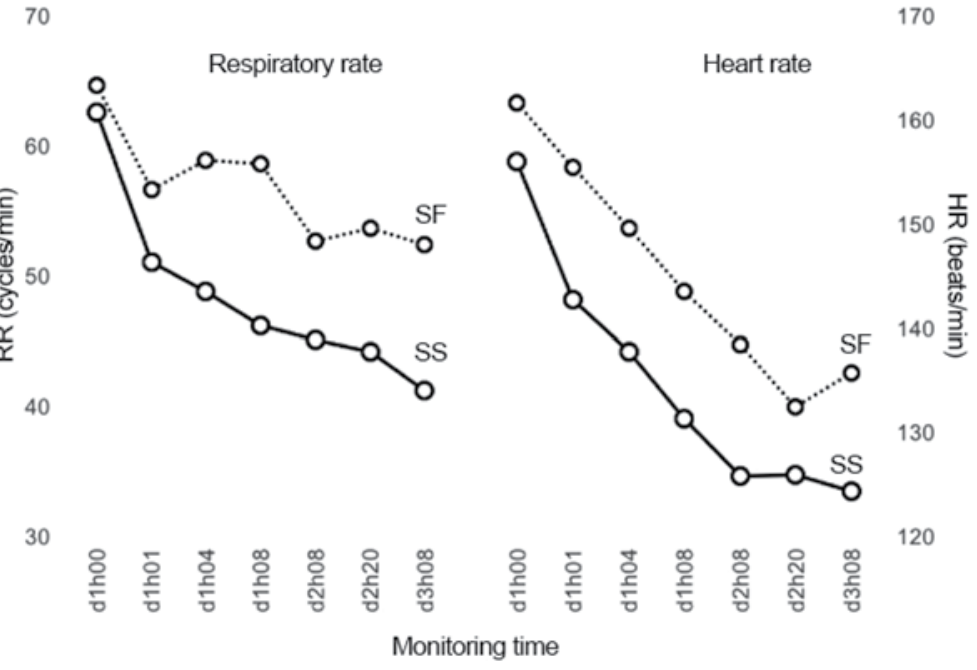

SS: support success; SF: support failure; RR: respiratory rate; HR: heart rate.

d1h00: day 1 hour 00, and so on. 
no data available for the 2014-2016 period at the units participating in this study for a more specific comparison.

In a retrospective study, Wing et al., ${ }^{6}$ compared 3 cohorts: the first cohort when HFNC was not available (2006); the second one when HFNC was available only at the PICU (2007-2008); and the third one when HFNC was available at the Emergency Department and the Pediatric Ward (2009). They included 848 patients and reported a reduction in the use of IMV from $16 \%$ to $8 \%$. They also observed an association between an earlier initiation of HFNC and a lower IMV requirement $(7.1 \%$ versus $18 \%)$, which was not seen in our analysis. Later, in 2013, Gonzales Martínez et al., reported a decrease in the admission rate to the PICU from $12.6 \%$ to $4.6 \%$, when HFNC was introduced in the general ward for patients with moderate-to-severe bronchiolitis, ${ }^{19}$ similar to what had been reported by Mayfield et al. (31\% versus $13 \%$ of admission to the PICU).${ }^{20}$ In a single-center, randomized study by Kepreotes et al., the comparison of LF oxygen use versus HFNC showed a decrease in SF (32\% versus $14 \%$ ), similar to what had been reported by Franklin et al. in a recently published multicenter, randomized trial, which found a reduction from $23 \%$ to $12 \% .{ }^{8,9}$

TABLE 4. Progression of respiratory rate in the patients with support success and failure

\begin{tabular}{|c|c|c|c|c|c|c|c|c|c|c|}
\hline \multirow[b]{2}{*}{ Moment } & \multicolumn{4}{|c|}{ Success } & \multicolumn{3}{|c|}{ Failure } & \multicolumn{3}{|c|}{ SS versus SF } \\
\hline & $\mathbf{n}$ & $\mathbf{R R}^{*}$ & $\begin{array}{l}\text { Intra-SS. } \\
\text { group diff }\end{array}$ & $p^{*}$ & $\mathbf{n}$ & $\mathbf{R R}^{*}$ & $\begin{array}{c}\text { Intra-SF } \\
\text { group diff. }\end{array}$ & $p^{\#}$ & $\begin{array}{c}\text { Diff. } \\
\text { between }\end{array}$ & $p^{\$}$ \\
\hline Pre-support & 189 & 63 (11.0) & & - & 18 & $65(9.1)$ & & & -2.1 & 0.3696 \\
\hline 1 hour & 187 & $51(10.9)$ & 10.8 & $<0.001$ & 18 & $57(10.4)$ & 7.9 & 0.002 & -5.6 & 0.0403 \\
\hline 4 hours & 178 & $48.7(12.1)$ & 2.5 & 0.003 & 17 & $59(12.2)$ & -1.2 & 0.639 & -10.1 & $<0.001$ \\
\hline 8 hours & 179 & $46.1(11.7)$ & 2.0 & 0.023 & 14 & 59 (10.7) & -2.0 & 0.496 & -12.5 & $<0.001$ \\
\hline 8:00 AM, day 1 & 178 & 45 (10.6) & 0.6 & - & 8 & $53(10.5)$ & 0.0 & - & -8.1 & \\
\hline 8:00 PM, day 1 & 171 & $44.1(10.9)$ & 1.8 & - & 8 & $54(13.2)$ & -4.7 & - & & \\
\hline 8:00 AM, day 2 & 169 & $41.2(10.3)$ & 0.5 & - & 6 & $52(13.4)$ & -3.0 & - & & \\
\hline
\end{tabular}

RR: respiratory rate; intra-SS group diff.: intra-support success group difference; intra-SF group diff.: intra-support failure group difference; diff. between: difference between the support success and support failure groups.

* mean and standard deviation.

$p \#: t$ test for paired samples.

$\mathrm{p} \$$ : $t$ test for independent samples.

FIGURE 2. Transition from one support method to the other in patients with support failure

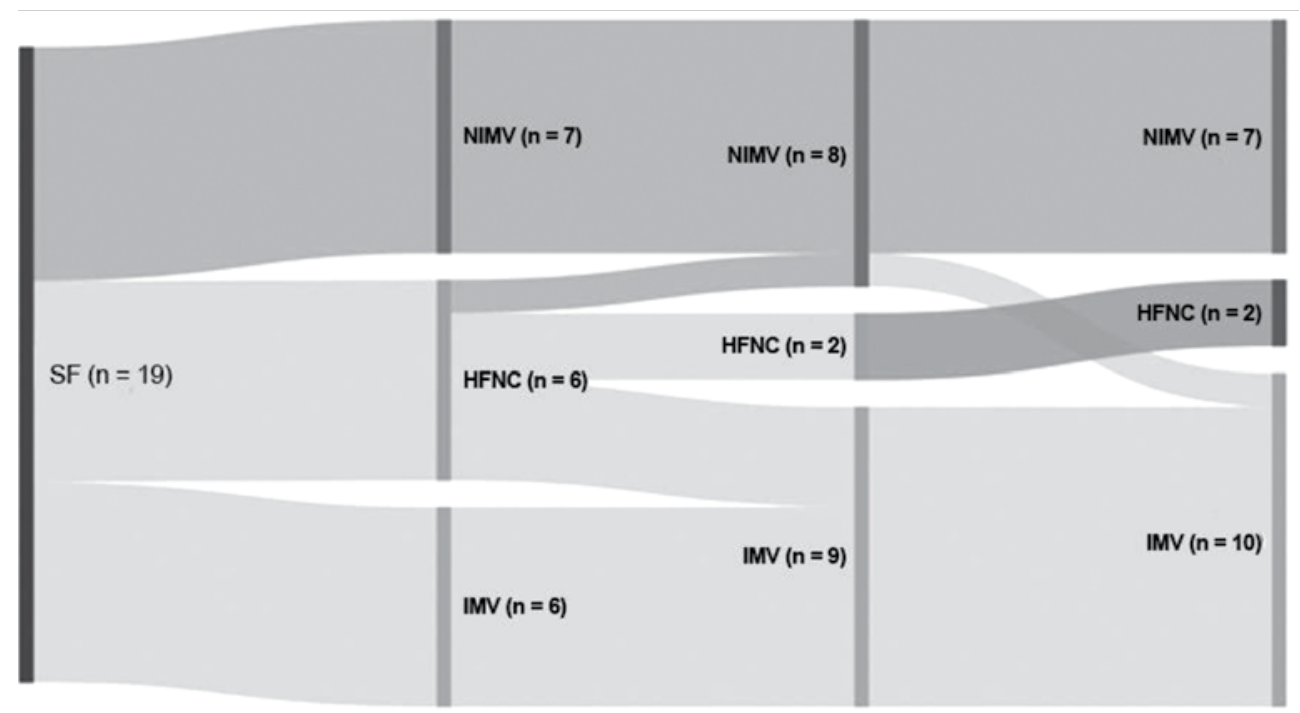

SF: support failure; NIMV: non-invasive mechanical ventilation; IMV: invasive mechanical ventilation;

HFNC: high-flow nasal cannula. 
In our study, we observed that the RR was a predictor of SS, which was consistent with the reports of other studies that found a RR reduction between 1 and 4 hours of treatment, which was significantly associated with SS. 11,19,21-25

Enteral nutrition was particularly relevant in the course of pediatric patients. To date, only 1 study reported, for 9 patients, the safety of feeding during treatment with HFNC. ${ }^{24}$ In our study, the treating teams did not contraindicate enteral feeding due to HFNC use. Although this information was not included in the protocol, informal comments from the investigators showed an homogeneous behavior in this regard.

As reported in the studies mentioned above, no adverse events were observed in association with HFNC.

Differences were found in some of the baseline patient characteristics among the 3 hospitals. Most likely, the differences in the universe of patients usually seen in these hospitals, from which the studied population was obtained, were due to each facility's profile and respective sphere of influence.

The main limitation of this study was that, since it was an observational study without a control group, it was not possible to establish if the reduction in the admission rate to the PICU was random or due to the presence of other factors beyond HFNC use. Therefore, it was decided to continue with prospective observation during 2018 to assess if such reduction was maintained.

\section{CONCLUSION}

The implementation of HNFC oxygen therapy under a protocol in this sample of patients diagnosed with ALRTI and hospitalized in the selected pediatric general wards resulted in a safe measure and no complications were recorded in association with its use. The reduction of the RR in the first hour of treatment was the most clear prognostic factor for therapeutic success. The patients with SF were transferred to the PICU or the intermediate care unit, and their course was not different from the usual.

\section{Acknowledgments}

Hospital Durand: Romina Carosella, Tomas Gilligan, Sabrina Guzmán, Silvia Piccinini, Ana Pota, Viviana Reines, Raúl Ruiz, Sandra Salzberg. Hospital Elizalde: Margarita Jara, Héctor Morales. Hospital Gutiérrez: Mónica Dastugue, Lucila de Luis, Miranda Fernández, Daniela Font, Camila García Laplaza, María Carolina Groisman,
Mónica Koch, Florencia Locatelli, María Victoria López, Normando Mascardi, Guadalupe Picón, Lucía Queizan, Natalia Rey, Sofía Rigou.

\section{REFERENCES}

1. Farias JA, Fernández A, Monteverde E, Flores JC, et al. Mechanical ventilation in pediatric intensive care units during the season for acute lower respiratory infection: a multicenter study. Pediatr Crit Care Med. 2012; 13(2):158-64.

2. Golubicki A, Gómez Traverso R. Observatorio de Enfermedades Respiratorias Agudas Bajas en Unidades de Internación Pediátricas. Ministerio de Salud de la Ciudad de Buenos Aires; 2015.

3. Comité Nacional de Neumonología, Comité Nacional de Infectología, ComitédeMedicina Interna. Recomendaciones para el manejo de las infecciones respiratorias agudas bajas en menores de 2 años. Resumenejecutivo. Arch Argent Pediatr. 2015; 113(4):373-4.

4. Dysart K, Miller TL, Wolfson MR, Shaffer TH. Research in high flow therapy: mechanisms of action. Respir Med. 2009; 103(10):1400-5.

5. Ten Brink F, Duke T, Evans J. High-Flow Nasal Prong Oxygen Therapy or Nasopharyngeal Continuous Positive Airway Pressure for Children With Moderate-to-Severe Respiratory Distress?* Pediatr Crit Care Med. 2013; 14(7):e326-31.

6. Wing R, James C, Maranda LS, Armsby CC. Use of high-flow nasal cannula support in the emergency department reduces the need for intubation in pediatric acute respiratory insufficiency. Pediatr Emerg Care. 2012; 28(11):1117-23.

7. Metge P, Grimaldi C, Hassid S, Thomachot L, et al. Comparison of a high-flow humidified nasal cannula to nasal continuous positive airway pressure in children with acute bronchiolitis: experience in a pediatric intensive care unit. Eur J Pediatr. 2014; 173(7):953-8.

8. Franklin D, Babl FE, Schlapbach LJ, Oakley E, et al. A Randomized Trial of High-Flow Oxygen Therapy in Infants with Bronchiolitis. N Engl J Med. 2018; 378(12):1121-31.

9. Kepreotes E, Whitehead B, Attia J, Oldmeadow C, et al. High-flow warm humidified oxygen versus standard low-flow nasal cannula oxygen for moderate bronchiolitis (HFWHO RCT): an open, phase 4, randomised controlled trial. Lancet. 2017; 389(10072):930-9.

10. Schibler A, Pham TM, Dunster KR, Foster K, et al. Reduced intubation rates for infants after introduction of highflow nasal prong oxygen delivery. Intensive Care Med. 2011; 37(5):847-52.

11. McKiernan C, Chua LC, Visintainer PF, Allen H. High flow nasal cannulae therapy in infants with bronchiolitis. J Pediatr. 2010; 156(4):634-8.

12. Kawaguchi A, Yasui Y, deCaen A, Garros D. The Clinical Impact of Heated Humidified High-Flow Nasal Cannula on Pediatric Respiratory Distress. Pediatr Crit Care Med. 2017; 18(2):112-9.

13. TalA, BavilskiC, Yohai D, Bearman JE, et al. Dexamethasone and salbutamol in the treatment of acute wheezing in infants. Pediatrics. 1983;71(1):13-8.

14. Feudtner C, Feinstein JA, Zhong W, Hall M, et al. Pediatric complex chronic conditions classification system version 2: updated for ICD-10 and complex medical technology dependence and transplantation. BMC Pediatr. 2014;14:199.

15. Wasserstein RL, LazarNA. TheASA's Statement on p-Values: Context, Process, and Purpose. Am Stat. 2016; 70(2):129-33.

16. Kruschke JK. What to believe: Bayesian methods for data analysis. Trends Cogn Sci. 2010; 14(7):293-300. 
17. Stanton JM. Reasoning with Data: an Introduction to Traditional and Bayesian Statistics Using R. New York: Guilford Press; 2017.

18. Jamil T, Ly A, Morey RD, Love J, et al. Default "Gunel and Dickey" Bayes factors for contingency tables. Behav Res Methods. 2017; 49(2):638-52.

19. González Martínez F, González Sánchez MI, Rodríguez Fernández R. Impacto clínico de la implantación de la ventilación por alto flujo de oxígeno en el tratamiento de la bronquiolitis en una planta de hospitalización pediátrica. An Pediatr. 2013; 78(4):210-5.

20. Mayfield S, Bogossian F, O'Malley L, Schibler A. High-flow nasal cannula oxygen therapy for infants with bronchiolitis: Pilot study. J Paediatr Child Health. 2014; 50(5):373-8.

21. Kallappa C, Hufton M, Millen G, Ninan TK. Use of high flow nasal cannula oxygen (HFNCO) in infants with bronchiolitis on a paediatric ward: a 3-year experience. Arch Dis Child. 2014; 99(8):790-1.

22. Bressan S, Balzani M, Krauss B, Pettenazzo A, et al. Highflow nasal cannula oxygen for bronchiolitis in a pediatric ward: a pilot study. Eur J Pediatr. 2013; 172(12):1649-56.

23. Hough JL, Pham TMT, Schibler A. Physiologic effect of high-flow nasal cannula in infants with bronchiolitis. Pediatr Crit Care Med. 2014; 15(5):e214-9.

24. Milani GP, Plebani AM, Arturi E, Brusa D, et al. Using a high-flow nasal cannula provided superior results to lowflow oxygen delivery in moderate to severe bronchiolitis. Acta Paediatr. 2016; 105(8):e368-72.

25. Kelly GS, Simon HK, Sturm JJ. High-flow nasal cannula use in children with respiratory distress in the emergency department: predicting the need for subsequent intubation. Pediatr Emerg Care. 2013; 29(8):888-92. 


\section{ANNEX \\ USE OF HIGH-FLOW NASAL CANNULA. \\ Experience in the Autonomous City of Buenos Aires, 2017 Study group of acute respiratory disease in pediatrics}

\section{ADDITIONAL MATERIAL}

Work plan. The members of the health care team of the participating general hospitalization wards received theoretical-practical training from health care providers who were experienced on HFNC use; training had been designed by the individuals responsible for the protocol implementation. Based on available evidence, the HFNC system was used if LF oxygen therapy failed according to the criteria established in the original document.

Hospitalized patients with a diagnosis of ALRTI received treatment based on the management guidelines for patients with ALRTI developed by the Ministry of Health of the CABA. The selection algorithm for oxygen therapy support (Annex 1) was included in these guidelines and a printout was hanged in a visible place inside the participating general hospitalization wards and PICUs. Patient assessment and the decision to initiate HFNC oxygen therapy or to escalate treatment were at the treating team's criteria.

Conventional oxygen therapy was administered by nasal cannula or mask with an oxygen flow below $4 \mathrm{~L} / \mathrm{min}$, and the target $\mathrm{SaO}_{2}(\geq 94 \%)$ was maintained by pulse oxygen saturation. LF oxygen therapy weaning was performed in every patient who achieved the target $\mathrm{SaO}_{2}$ in ambient air.

HF oxygen therapy was administered using the Airvo 2® humidifier by Fisher \& Paykel Healthcare ${ }^{\circledR}$ with an Optiflow ${ }^{\circledR}$ infant cannula (up to $20 \mathrm{~L} / \mathrm{min}$ ) or an Optiflow ${ }^{\circledR}$ pediatric cannula (up to $25 \mathrm{~L} / \mathrm{min}$ ). The Airvo 2® is a humidifier system with integrated flow generator that allows delivering HF oxygen at an optimal temperature and relative humidity with a previously set flow and $\mathrm{FiO}_{2}$. The integrated flow generator allows delivering a flow between 2 and $60 \mathrm{~L} / \mathrm{min}$. Oxygen was obtained from the standard wall oxygen supply (not compressed), and an integrated ultrasonic oxygen analyzer warranted the desired $\mathrm{FiO}_{2}$ delivery. A tube with a heater cable and temperature sensor to measure the delivered gas was connected to the flow generator and the selected cannula. The cannula was selected based on the patient's weight, trying not to obstruct more than $50 \%$ of the nostrils. Flow was initially set at $2 \mathrm{~L} / \mathrm{kg} /$ min. $\mathrm{FiO}_{2}$ was initially set at 0.9 and rapidly lowered to less than 0.6 once the patient was stable, with a target $\mathrm{SaO}_{2}$ of $94 \%$ in a period of not more than 3 hours. $\mathrm{FiO}_{2}$ was reduced by 0.05 -point intervals, $\mathrm{SaO}_{2}$ was adjusted to $94 \%$ by pulse oxygen saturation, and the flow was kept consistently at $2 \mathrm{~L} / \mathrm{kg} /$ min. If $\mathrm{SaO}_{2}$ fell below $94 \%$ with the reduction of $\mathrm{FiO}_{2}$, the latter was increased back to the previous level. HFNC weaning was performed in every patient who was able to remain at least 4 hours with a $\mathrm{FiO}_{2}$ of 0.21 .

The protocol did not contain any specification in relation to patient feeding, which was left at the treating team's criteria.

A responsible investigator was designated at each hospitalization unit, who was in charge of recording all the data to be included in the protocol forms.

SS was defined if the patient required only HFNC oxygen therapy, whereas SF was considered if the patient required an additional treatment: 1) transfer to the PICU or intermediate care unit, 2) change of ventilatory support to NIMV or IMV.

Data collection: the data collection forms were specially designed to this end by the study coordinating team (Annex 2). The data recorded in the paper forms were then introduced in an online form designed using JotForm (www.jotform.com) by the same coordinating team. Access to the online forms was restricted using individual users and passwords, which were protected under the responsibility of each principal investigator. Once introduced, these data were available for their review and analysis by the study coordinators, who monitored them in real time. In these forms, each individual patient was identified using a unique code assigned by each site. The link between the patient code and personal identifying data was safeguarded at each participating hospital, who committed to prevent its disclosure by virtue of the terms of Act no. 25326 for the Protection of Personal Information. 